\section{Effect of One and Two Split Aerobic Exercise Sessions on Circadian Rhythm of Heart Rate and Blood Pressure}

\author{
Saeed Nikookheslat ${ }^{1}$, Vahid Sar Sarraf ${ }^{1^{*}}$ and Mahmoud Ahmadzadeh ${ }^{2}$ \\ ${ }^{1}$ Sport Science Faculty, University of Tabriz, Iran
}

${ }^{2}$ Exercise Physiology, University of Tabriz, Iran

*Corresponding author: Vahid Sar Sarraf, Sport Science Faculty, University of Tabriz, Iran, Tel: +989382138861; E-mail: Spaniol_man@yahoo.com

Received date: April 25, 2016; Accepted date: March 06, 2017; Published date: March 13, 2017

Copyright: (c) 2017 Nikookheslat S, et al. This is an open-access article distributed under the terms of the Creative Commons Attribution License, which permits unrestricted use, distribution and reproduction in any medium, provided the original author and source are credited.

\begin{abstract}
The present study investigated the circadian rhythm variations of heart rate and blood pressure due to 1 (60 minutes) and 2 split exercise sessions $(2 \times 30$ minutes) a day. Fourteen healthy students from the University of Tabriz volunteered to participate in current study. Two groups of subjects in a crossover design were randomly allocated as group I (one session per day, twice a day) and vice versa for group II. Having completed the test (treadmill running with an intensity of 70 per cent of Maximum Heart rate), heart rate and blood pressure (both systolic and diastolic) were measured every two hours for 24 hours. Repeated measure (ANOVA) was used for analysis. Each of variables showed normal circadian rhythm. Compared to two split exercise sessions a day, one session a day demonstrated that the level of heart rate circadian rhythm has been set at a rate of five percent below. Circadian rhythm of systolic and diastolic blood pressure in the two split sessions compared with one session a day tended to be set at a lower level and higher level respectively. One aerobic exercise session compared to two split sessions a day with the same total time of exercise a day may decrease systolic blood pressure and heart rate within 24 hours following aerobic treadmill running exercise. Split exercise session could be an appropriate approach for increasing more calories and lowering blood pressure among health seekers while it may delay recovery for the next day training or competition for professional athletes.
\end{abstract}

Keywords: Circadian rhythm; Heart rate; Blood pressure; Aerobic treadmill running; Split exercise sessions

\section{Introduction}

Circadian rhythms and their regulations specifically by exercise are a basic quality of human physiology, which have extensively been scrutinized in sport field [1-3]. It is commonly known that, in healthy humans, heart rate (HR) and blood pressure (BP) along increase during the day and decrease during the night, as a result of sleep-wake or rest-exercise changes [1,4]. It is difficult to describe circadian rhythms in variables relevant to sports and exercise, while at the same time provide information on their underlying mechanisms [5] Monitoring circadian rhythm variations of physiological variables due to exercise is of utmost importance concerning the cardiovascular health status of various populations in a society (Health classification, age groups, gender differences, chronic diseases, endocrinology etc.) [6-8].

Nowadays, split exercise sessions are becoming more and more popular among coaches and athletes to put out the flames of questions laid down on recovery, oxygen consumption, weight control and cardiovascular variables such as heart rate and blood pressure [9-13]. Knowledge of the time course of variables dependent to physiological modifications (of heart rate and blood pressure) induced by split aerobic exercise sessions is important in various sports from which running is the most popular choice among individuals. This is true in both the professional field, identifying the most favorable split aerobic exercise sessions, which leads to maximum result from performance, and for the amateurs, allowing person to take full advantage of the benefits, which split aerobic exercise sessions, produces for the body [9,12-15].

On the other hand, investigations on circadian rhythm variation due to exercise refers to studies in the 1970s on NASA astronauts who evaluated the effect of physical exercise on the time cycles of physiological variables, such as HR and other electrocardiogram parameters. At that time, studies examined the effects of physical exercise, feasible in a spacecraft, on the circadian structure of the subjects and consequently on their well-being [16]. In normotensive subjects, Heart rate and blood pressure both exhibit a strong circadian pattern with values for blood pressure and heart rate typically peaking in the early morning period whereas it shows a weak circadian pattern among clinical subjects [17]. This appears to be mediated by autonomic nervous system which itself is under regulatory control of the Suprachiasmatic nucleus of hypothalamus - 'the master oscillator'. This master oscillator also generates circadian rhythmic patterns for other biological events. It is increasingly being appreciated that many diseases have a pattern associated with circadian rhythms of various biological functions of the body, more so the cardiovascular diseases. The existence of circadian rhythms in heart rate and blood pressure means that the rate-pressure product would also show a similar rhythm [18]. It would be interesting to give comprehensive and practical information, suitable for everyone (ranging from athletes to healthy individuals) who is seeking a useful exercise planning according to their goals (faster recovery or weight control and increasing body metabolic rare). No sooner had investigations on the on circadian rhythm variations of heart rate and blood pressure (systolic and diastolic pressure) been considered than we noticed rarely there could be found similar studies since investigating split exercise sessions (one compared with two sessions a day) and circadian rhythm 
Citation: Nikookheslat S, Sarraf VS, Ahmadzadeh M (2017) Effect of One and Two Split Aerobic Exercise Sessions on Circadian Rhythm of Heart Rate and Blood Pressure. J Sports Med Doping Stud 7: 189. doi:10.4172/2161-0673.1000189

Page 2 of 5

variations have been running on its infancy [19]. Concerning the importance of cardio-vascular parameters mainly including heart rate, blood pressure and their variations due to aerobic exercise from aspect of chronobiology, current study aimed at latter to identify the effect of split exercise sessions (one bout of 60 minutes Vs. two bouts of 30 minutes aerobic exercise) on circadian rhythms [17] among normotensive young males.

\section{Participants and Methods}

\section{Participants}

In order to put the research into practice, 12 healthy young males, aged $23.2 \pm 1$ years (mean \pm standard deviation), with Vo2max $49.2 \pm$ $1.3 \mathrm{mlo}^{2} \cdot \mathrm{min}^{-1} \cdot \mathrm{kg}^{-1}$ and with Body Mass Index of $21.98 \pm 0.3 \mathrm{~kg} \cdot \mathrm{m}^{-2}$ volunteered to participate.

Then, they were randomly divided two groups. All participants were medically examined and there were no history of cardiovascular diseases and smoking and did not take any drugs. They did not engage in any physical activity except the protocol of current research and most importantly, they had not had any flight beyond time zone during 6 months before conducting study if had, it would have affected circadian rhythms. The study was approved by the university ethics committee and all participants gave their consent inform.

Participants attended the laboratory and their Vo2max was determined via exhaustive treadmill running protocol (Bruce protocol) and other descriptive data including weight, height and BMI. Having familiarized with the study design, participants were instructed to refrain from any exercise and physical activity except protocol exercise and not to take drugs and not to smoke or drink alcohol and foods or drinks containing caffeine. The pre-exercise systolic and diastolic blood pressures were of $123.0 \pm 7.4$ and $86.5 \pm 3.2 \mathrm{mmHg}$ respectively. During the first attendance of participants in laboratory, anthropometric measurements of height, weight, Body Mass Index (BMI), Vo2max, Maximum Heart Rate (MHR) and resting blood pressure (using a mercury sphygmomanometer) were obtained. Participants were familiarized with the experimental protocols, pretest protocol (Bruce protocol) all of which were performed on treadmill.

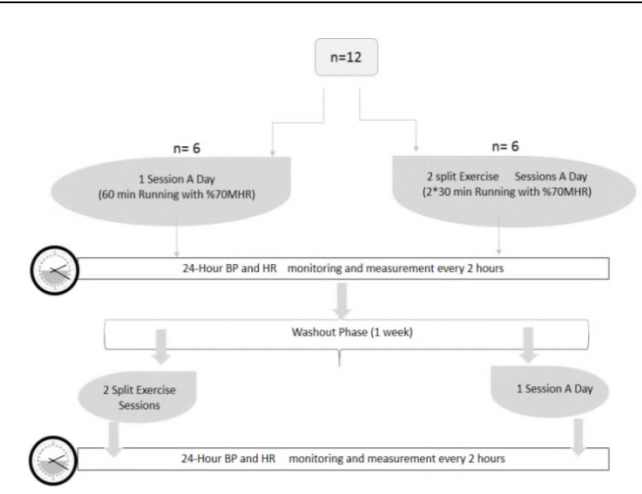

Figure 1: Study design.

\section{Study Design}

All participants were under direct monitoring for 3 hours before and 24 hours after completing experimental protocols. Having familiarized with the research procedure (Figure 1), participants attended laboratory to perform the experimental procedure. The experimental procedure consisted of two different protocols: The first protocol included one bout of $60 \mathrm{~min}$ treadmill running with the intensity of 60 per cent Vo2max; the second one included two bouts of 30 min treadmill running with the intensity of 60 per cent Vo2max (Split Exercise Sessions) (Figures 2-4).
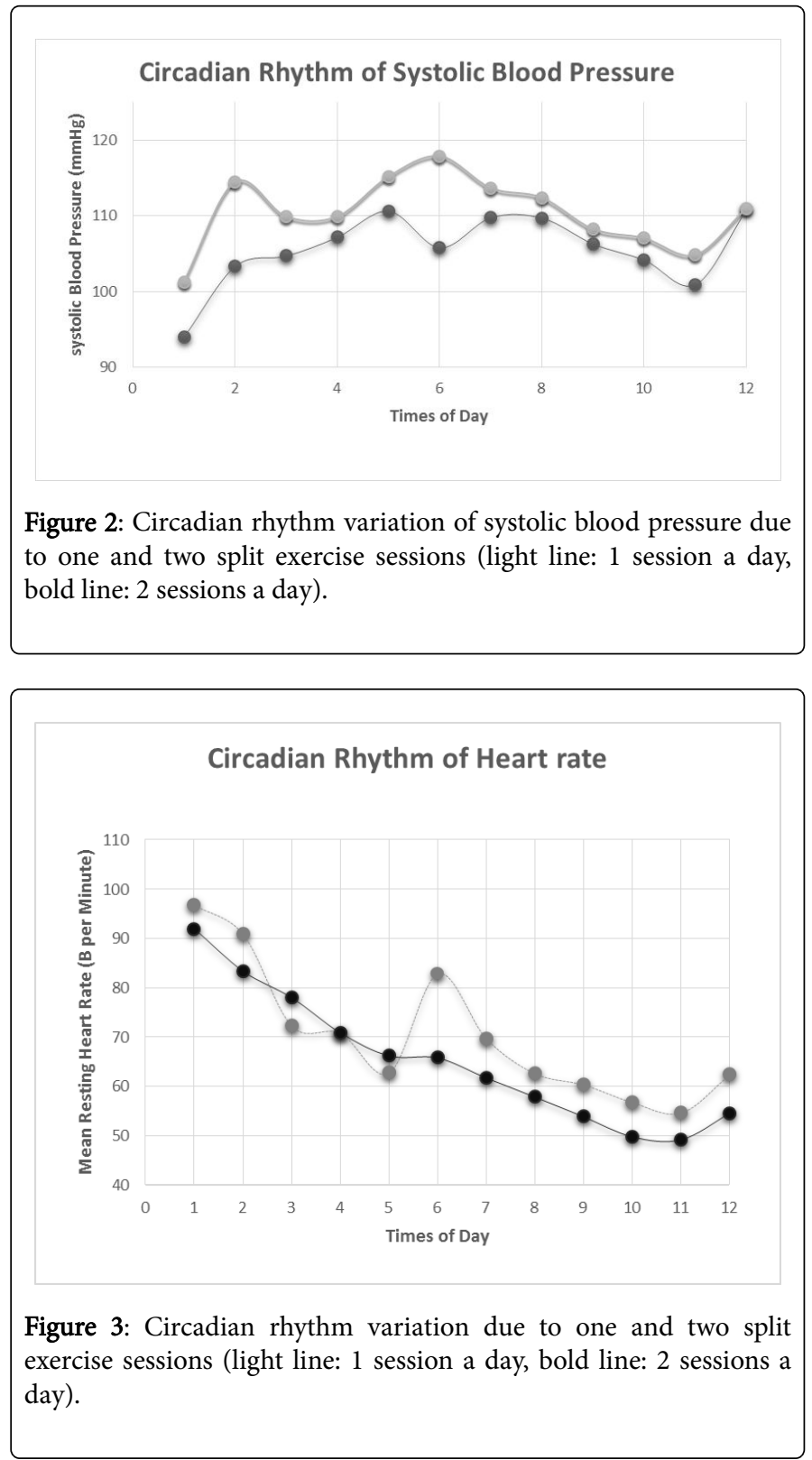
Citation: Nikookheslat S, Sarraf VS, Ahmadzadeh M (2017) Effect of One and Two Split Aerobic Exercise Sessions on Circadian Rhythm of Heart Rate and Blood Pressure. J Sports Med Doping Stud 7: 189. doi:10.4172/2161-0673.1000189

Page 3 of 5

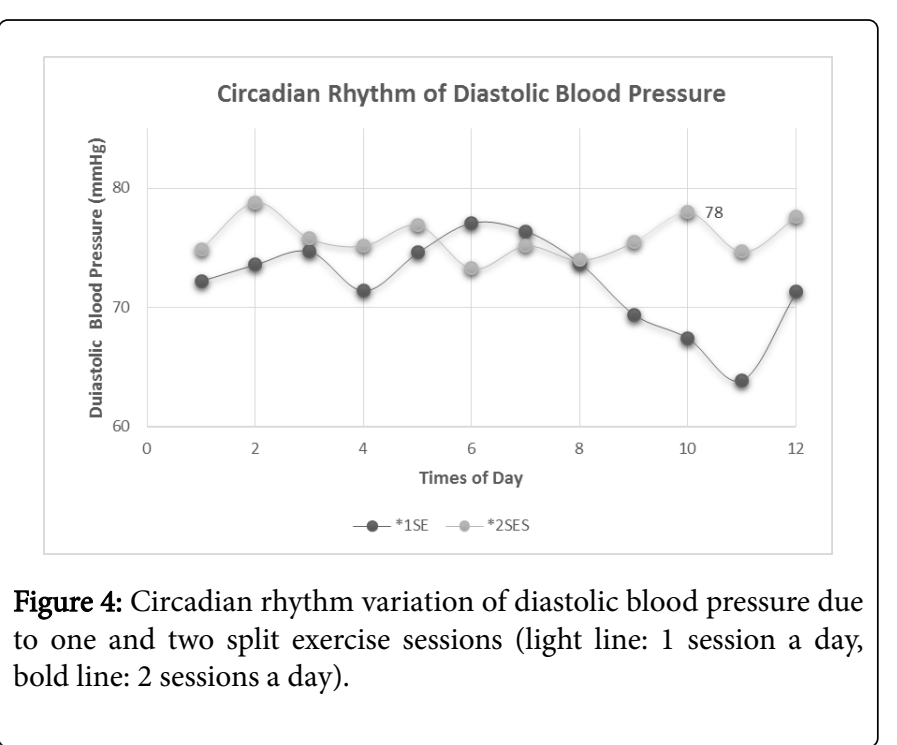

\section{Data Collection}

The experimental protocols (one and two split exercise sessions), all were conducted in Tabriz university gym wherein light levels at approximately 200 lux, and temperature at $20^{\circ} \mathrm{C}$ were controlled. Pre exercise $\mathrm{BP}$ and $\mathrm{HR}$ were measured using mercury sphygmomanometer and HR monitor polar. Resting BP and HR both were measured for 3 times at each time of day throughout the study and mean values were obtained to put in the analysis and statistical process (Tables 1-3).

Several studies have conducted to identify the reliability of mercury sphygmomanometer for accurately measuring $\mathrm{BP}$ in medical science and medical research. Participants were allowed to stop exercise or continuing the experimental procedure whenever they wanted. One and two bouts of exercise a day were performed with 3 days apart. Participants were divided into 2 groups (cross over design).

The first group was supposed to complete one bout of exercise in a day, then take rest for the next 3 days, and eventually perform two bouts of exercise in a day. The second group was supposed to complete the other way around what went through in first group (two bouts of exercise a day and after 3 days being apart, performed one bout of exercise). Following a $10 \mathrm{~min}$ warm-up, participants based on their group procedure began to complete the test. Monitoring the intensity of exercise were observed via the polar and 60 percent of MHR which all had been obtained in the first attendance of participants to measure Vo2max. The first group began to perform one bout of exercise (1B) for $60 \mathrm{~min}$ at 08:30 AM and finished at 09:30 AM. Then, they took rest in supine position for $15 \mathrm{~min}$ before $\mathrm{BP}$ and $\mathrm{HR}$ recording and the second group began two bouts of exercise (2B) for $30 \mathrm{~min}$ at 09:00 $\mathrm{AM}$ and finished at 09:30 $\mathrm{AM}$ and rested in supine position for $15 \mathrm{~min}$ before $\mathrm{BP}$ and $\mathrm{HR}$ recording.

The 24 hour BP and HR monitoring began from 10:00 AM up to the subsequent day till 10:00 AM. BP (both systolic and diastolic blood pressure), HR (via polar which was fitted to participants chest throughout the study) were recorded as the average obtained from 3 times measurements every 2 hours following exercise at the times of $10: 00,12: 00,14: 00,16: 00,18: 00,20: 00,22: 00,24: 00,02: 00,04: 00,06: 00$, $08: 00,10: 00$. No participants were allowed to engage in a moderate or heavy physical activity nor exercise and all were under direct observation by researchers in controlled environment (light and temperature). They were supposed to go to their bed at 10 oclock and get up from their bed at 06:00 o'clock. Participants in two split exercise sessions (according their group procedure) were intervened by second bout of exercise at 07:00 for $30 \mathrm{~min}$, finished their exercise at 07:30, and prepared to resume their 24 monitoring BP and HR for the rest of remained time phase. The second bout of $30 \mathrm{~min}$ exercise was performed exactly the way the first bout of $30 \mathrm{~min}$ exercise was performed at 09:00 to 09:30. All measurements were obtained in supine position and with $15 \mathrm{~min}$ rest before measurements. Diet control, food and fluid intakes were according to the diet plan for a healthy man and isocaloric as well as the exercise protocols (60 min vs. $2 \times 30$ min both with $\% 60$ Vo2max intensity) [20].

BP (systolic and diastolic) and HR values were recorded after calculating the average value of three times of repetitive measuring and therefore double product (DBPP) calculated as HR (beat.min-1) multiplied by systolic blood pressure $(\mathrm{mmHg})$. By considering the relationship between HR and systolic BP, DBPP has also a circadian rhythm throughout that 24-hour a day. DBPP values were recorded the same as HR and BP every 2 hours following experimental exercises from 10:00 (first time of day following exercise) up to next day at 10:00.

\begin{tabular}{|l|l|l|l|l|l|l|l|l|l|l|l|l|}
\hline & $10: 00$ & $12: 00$ & $14: 00$ & $16: 00$ & $18: 00$ & $20: 00$ & $22: 00$ & $24: 00: 00$ & $02: 00$ & $04: 00$ & $06: 00$ & $08: 00$ \\
\hline *1SE & 96.71 & 90.86 & 72.29 & 70.86 & 62.79 & 82.93 & 69.71 & 62.64 & 60.36 & 56.79 & 54.64 & 62.43 \\
\hline **2SES & 91.93 & 83.36 & 78 & 70.86 & 66.21 & 65.79 & 61.71 & 57.86 & 53.86 & 49.79 & 49.21 & 54.57 \\
\hline
\end{tabular}

Table 1: Mean values of heart rate (beats per minute) in 12 times of day.

\begin{tabular}{|l|l|l|l|l|l|l|l|l|l|l|l|l|}
\hline & $10: 00$ & $12: 00$ & $14: 00$ & $16: 00$ & $18: 00$ & $20: 00$ & $22: 00$ & $24: 00: 00$ & $02: 00$ & $04: 00$ & $06: 00$ & $08: 00$ \\
\hline *1SE & 93.93 & 103.36 & 104.71 & 107.21 & 110.64 & 105.79 & 109.79 & 109.71 & 106.29 & 104.14 & 100.93 & 110.86 \\
\hline **2SES & 101.29 & 114.5 & 109.93 & 109.93 & 115.21 & 117.93 & 113.71 & 112.43 & 108.29 & 107.07 & 104.86 & 111 \\
\hline
\end{tabular}

Table 2: Mean values of systolic blood pressure $(\mathrm{mmHg})$ in 12 times of day. 
Citation: Nikookheslat S, Sarraf VS, Ahmadzadeh M (2017) Effect of One and Two Split Aerobic Exercise Sessions on Circadian Rhythm of Heart Rate and Blood Pressure. J Sports Med Doping Stud 7: 189. doi:10.4172/2161-0673.1000189

Page 4 of 5

\begin{tabular}{|l|l|l|l|l|l|l|l|l|l|l|l|l|}
\hline & $10: 00$ & $12: 00$ & $14: 00$ & $16: 00$ & $18: 00$ & $20: 00$ & $22: 00$ & $24: 00: 00$ & $02: 00$ & $04: 00$ & $06: 00$ & $08: 00$ \\
\hline *1SE & 72.2 & 73.6 & 74.7 & 71.4 & 74.6 & 77.1 & 76.4 & 73.7 & 69.4 & 67.4 & 63.9 & 71.3 \\
\hline **2SES & 74.9 & 78.8 & 75.8 & 75.2 & 76.9 & 73.3 & 75.2 & 74 & 75.5 & 78 & 74.7 & 77.6 \\
\hline \multicolumn{10}{|l|}{ *1 Session Exercise and **2 Split Exercise Sessions } \\
\hline
\end{tabular}

Table 3: Mean values of diastolic blood pressure $(\mathrm{mmHg})$ in 12 times of day.

\section{Statistical analyses}

Data were analyzed using SBPPSS 17. Descriptive statistic including mean and standard deviation were calculated. Klomogorov - Smirnof test was used to test the homogeneity of variance. The statistical significance of exercise-induced variations on circadian rhythms was assessed using repeated measure ANOVA. Tuckey post hoc test was used to compare different means. Statistical significance for all tests was set at $\mathrm{P}<0.05$.

\section{Results}

After the subjects completed the two protocols $\left(1^{\star} 60\right.$ minute running and $2^{\star} 30$ minute running), values of HR and BP (SBP and $\mathrm{DBP})$ every 2 hour were measured. Results showed that, there were significant variations between circadian rhythms of HR $(\mathrm{P}=0.03)$, Systolic Blood Pressure (SBPP) $(\mathrm{P}=0.009, \mathrm{~F}=7.85)$ followed by single bout of exercise $(60 \mathrm{~min})$ and split exercise sessions $(30 \mathrm{~min}$ for each bout) $(\mathrm{P}<0.05)$ regarding the whole times of day (12 times of day). There were not significant variations in circadian rhythm of double product (DBPP) and Diastolic Blood Pressure (DBPP) between single bout and two bouts of exercise.

As to HR circadian rhythm followed by split-exercise sessions in Figure 3, there was no significant difference in mean \pm SD resting HR from 10:00 (first time of day) to 18:00 (fifth time of day) (91.9 \pm 10.9 $\mathrm{mmHg}$ vs. $96.7 \pm 6.8 \mathrm{mmHg}$ in one and two sessions respectively). But, after sixth time of day (20:00) which is followed by second bout of 30 min exercise, there is a significant difference when compared with the single bout exercise $(83.0 \pm 1.8 \mathrm{mmHg}$ vs. $65.8 \pm 2.2 \mathrm{mmHg})$. During the rest of the times of day, there is no significant difference between sing and two bouts of exercise although HR circadian rhythm of two bouts tends to be laid above that of single bout after 20:00 up until 10:00 (20:00, 22:00, 00:00, 02:00, 04:00, 06:00, 08:00, 10:00).

Considering circadian rhythm of SBPP, there is a significant difference between single bout $(117.6 \pm 10.5 \mathrm{mmHg})$ and two bouts $(105.6 \pm 5.2 \mathrm{mmHg})$ at 20:00 and 22:00 (sixth, seventh times of day) with $10.2 \%$ decrease in SBPP followed by two bouts of exercise. There were no significant differences between the rests of other times of day. Overall mean \pm standard deviation of SBPP circadian rhythm in single bout and two bouts of exercise are $101.3 \pm 12.5 \mathrm{mmHg}$ and $93.9 \pm 4.5$ mmHg.

Circadian rhythm of DBPP did not show any variation in twelve times of day when followed by single bout and two bouts of exercise (73.0 \pm 3.5 and $77.0 \pm 2.8$ in single bout and two bouts of exercise respectively). Circadian rhythm of DBPP did not demonstrate any variation in twelve times of day although DBPP of 6 times of day were slightly higher in two bouts when compared with the single bout $(20: 00,22: 00,24: 00,02: 00,04: 00,06: 00,08: 00)$. The mean \pm SD of
DBPP in single and two bouts of exercise were $7204.6 \pm 1476.2 \mathrm{U}$ and $7387.7 \pm 1215.8 \mathrm{U}$ respectively.

\section{Discussion}

We explored variations occurred in the circadian rhythms of HR, SBP and DBP due to one exercise session (1ES) (60 min) and two split aerobic exercise sessions (2SES) $(2 \times 30 \mathrm{~min})$. The results of present study show that there is a significant difference between 1ES and 2SES when time of day changes are considered (for instance 10:00 in 1ES is compared with 10:00 in 2SES and so on). Circadian rhythm variation of all variables in present study may not be firmly indicated since the changes are merely seen up to 4 hours after the exercise finished. The HR circadian rhythm in both protocols demonstrated normal fluctuation, which is consistent with results of Carandente et al. that studied effect of morning and evening exercise on heart rate circadian rhythm [21]. They found that all subjects had normal fluctuation within 24 hour a day. Carandente also stated that the highest values for heart rate were observed following morning exercise session, which is consistent with our finding. Since the participants were healthy and without any history of chronic and/or acute diseases, it was expected to show such normal pattern. In addition, BP revealed a normal fluctuation within 24 hours a day during which SBP reached a peak at 20:00 and 08:00 in 1ES and 2SES respectively. The lowest SBPP values detected at 04:00 in both protocols. This finding is consistent with that of Shea et al. that stated the highest values for BP could be found in the evening (21:00). They suggested that there exists a robust endogenous circadian rhythm in BP. They stated that the endogenous BP rhythm is unlikely to underlie the well-documented morning peak in adverse cardiovascular events [22]. Values for DBP were not consistent with Shea et al. finding due to difficulties in measuring diastolic pressure, which have always been with controversies [23].

With regard to current study, there are two important findings. First, it could be detected that circadian rhythm tends to pursue its path despite the exercise intervention within 24 hours. Although significant interventions in synchronicity after up to 4 hours following exercise could be detected, it resumes its path to retain resting homeostasis. Physiological system always controls this pattern because it is vital from aspect of adaptations to environmental changes (time of day, day and night, season changes, temperature and etc.). Second, we may better be able to distribute the exercise activity duration in morning and evening if the goal is to manage the BP (keeping in normal range). The latter finding can be used for athletes and coaches too. If the goal is to accelerate the recovery, 2 sessions training a day may not be rational. Additionally, worth to be indicated, explorations in current study into the influence of single bout and two bouts of exercise on circadian rhythm may be one of the pioneering studies which recently has been conducted along with its own associated difficulties. In parallel with the evidence obtained from our own data, there are numerous studies conducted to investigate the interaction of exercise and HR, BP and 
DBPP circadian rhythms although the approaches adopted in current research were of innovation and creative methods. Rarely there could be found researches in which bouts of exercise are discussed in association with circadian rhythms (mainly HR, BP and DBPP). Several studies have investigated circadian variations of various body systems. Circadian variation in some cardiovascular parameters such as cardiac output during exercise has yet to be identified [24]. Other variables such as systolic, diastolic blood pressure and heart rate tend to exhibit circadian rhythmicity, although there are difficulties measuring such parameters with the required accuracy under exercise conditions. Cohen et al. measured heart rate at rest, during exercise on a rowing ergometer, and in the recovery period of this exercise at seven times of the solar day with the lowest heart rates occurring between 04:00 and 08:00 h. This temporal pattern was evident both during and after exercise. Other studies using an incremental exercise task have demonstrated the heart rate irrespective just prior to exhaustion, when exercise intensity is maximal, does not vary with time of day [25]. Such discrepancies might be related to a reduction in the range of circadian variability with increasing levels of exercise or a failure to detect the circadian rhythm as the ceiling of physiological capability is reached during the exercise test $[24,26]$.

Findings of current study put forward practical implications in general for healthy people to control their cardiovascular (CV) health and in particular for coaches and athletes to manage their training sessions and bouts near to competition, during preparatory season and recovery. Statistics showed that there are variations in circadian rhythms of heart rate, SBP without significant variation in DBP. It is also should be mentioned that SES prior to competitions or intensive training may not be an appropriate training session since it can significantly increase post-exercise caloric expenditure and based on current research, this can be concluded by measuring heart rate. Unlikely, SES could be an appropriate alternative for those who are seeking health and weight control [26].

\section{Conclusion}

Split exercise session could be an appropriate approach for increasing more calories and lowering blood pressure among health seekers while it may delay recovery for the next day training or competition for professional athletes.

\section{References}

1. Biaggioni I (2008) Circadian clocks, autonomic rhythms, and blood pressure dipping. Hypertension 52: 797-798.

2. Scheer FA, Shea SA (2014) Human circadian system causes a morning peak in prothrombotic plasminogen activator inhibitor-1 (PAI-1) independent of the sleep/wake cycle. Blood 123: 590-593.

3. Farah BQ, Ritti-Dias RM, Balagopal P, Hill JO, Prado WL (2014) Does exercise intensity affect blood pressure and heart rate in obese adolescents? A 6-month multidisciplinary randomized intervention study. Pediatr Obes 9: 111-120.

4. Clark L, Denby L, Pregibon D, Harshfield GA, Pickering TG, et al. (1987) A quantitative analysis of the effects of the activity and time of day on the diurnal variations of blood pressure. J Chronic Dis 40: 671-681.

5. Edwards BJ, Waterhouse J, Atkinson G, Reilly T (2002) Exercise does not necessarily influence the phase of the circadian rhythm in temperature in healthy humans. J Sports Sci 20: 725-732.
6. Pagonas N, Dimeo F, Bauer F, Seibert F, Kiziler F, et al. (2013) The impact of aerobic exercise on blood pressure variability. J Hum Hypertens 28: 367-371.

7. Tjønna AE, Lee SJ, Rognmo O, Stølen TO, Bye A, et al. (2008) Aerobic interval training versus continuous moderate exercise as a treatment for the metabolic syndrome: A pilot study. Circulation 118: 346-354.

8. Ruwald MH, Moss AJ, Jons C, McNitt S, Ruwald AC, et al. (2013) Circadian rhythm variation of malignant ventricular arrhythmias and its relation to mortality among patients with mild heart failure. Circulation 128: A17451.

9. Larsen I, Welde B, Martins C, Tjønna (2014) High-and moderateintensity aerobic exercise and excess post-exercise oxygen consumption in men with metabolic syndrome. Scand J Med Sci Sports 24: e174-e179.

10. Kang J, Mangine GT, Ratamess NA, Faigenbaum AD, Hoffman JR (2014) Acute effect of intensity fluctuation on energy output and substrate utilization. J Strength Cond Res 28: 2136-2144.

11. Soares-Miranda L, Sattelmair J, Chaves P, Duncan GE, Siscovick DS (2014) Physical activity and heart rate variability in older adults: The cardiovascular health study. Circulation 129: 2100-2110.

12. Del Gobbo L, Kalantarian S, Imamura F, Lemaitre R, Siscovick D, et al. (2014) Contribution of preventable risk factors for incident congestive heart failure in older adults: The cardiovascular health study. Circulation 129: AMP47.

13. Marsden DL, Dunn A, Callister R, Levi CR, Spratt NJ (2013) Characteristics of exercise training interventions to improve cardiorespiratory fitness after stroke a systematic review with metaanalysis. Neurorehabil Neural Repair 27: 775-788.

14. Atkinson G, Reilly T (1996) Circadian variation in sports performance. Sports Med 21: 292-311.

15. Bagger M, Petersen PH, Pedersen PK (2003) Biological variation in variables associated with exercise training. Int J Sports Med 24: 433-440.

16. Veerman DBPP, Imholz BP, Wieling W, Wesseling KH, van Montfrans GA (1995) Circadian profile of systemic hemodynamic. Hypertension 26: 55-59.

17. Liu S, Thomas SG, Sasson Z, Banks L, Busato M, et al. (2013) Blood pressure reduction following prolonged exercise in young and middleaged endurance athletes. Eur J Prev Cardiol 20: 956-962.

18. Nene SB, Sumandatta AR, Praveenya SV, Lavanya K (2013) Chronobiology of rate pressure product in young adults. Int J Sci Res 2: 86-89.

19. O’Connor P, Youngstedt S, Buxton O, Brues M (2004) Air travel in sports federation Interna-tionale de Medecine du sport position statement.

20. Kaminsky LA, Padjen S, LaHam-Saeger J (1990) Effect of split exercise sessions on excess post-exercise oxygen consumption. Br J Sports Med 24: 95-98.

21. Carandente F, Montaruli A, Roveda E, Calogiuri G, Michielon G, et al. (2006) Morning or evening training: effect on heart rate circadian rhythm. Sport Sci Health 1: 113-117.

22. Shea SA, Hilton MF, Hu K, Scheer FA (2011) Existence of an endogenous circadian blood pressure rhythm in humans that peaks in the evening. Circ Res 108: 980-984.

23. Lim YH, Shin J, Choi SY, Oh KW, Kim Y (2013) Epidemiologic factors associated with errors in blood pressure measurements obtained with an automated device compared with a mercury Sphygmomanometer. Eur Heart J 34: P5643.

24. Reilly T, Brooks GA (1986) Exercise and the circadian variation in body temperature measures. Int J Sports Med 7: 358-362.

25. Cohen CJ (1980) Human circadian rhythms in heart rate response to a maximal exercise stress. Ergonomics 23: 591-595.

26. Winget CM, Beljan JR (1980) Circadian systems in medicine. Nebr Med J 65: 303-306. 